\title{
Novel electric field effects on Landau levels in Graphene
}

\author{
Vinu Lukose $*$ R. Shankar \\ The Institute of Mathematical Sciences, \\ C.I.T. Campus, Chennai 600 113, India
}

\begin{abstract}
A new effect in graphene in the presence of crossed uniform electric and magnetic fields is predicted. Landau levels are shown to be modified in an unexpected fashion by the electric field, leading to a collapse of the spectrum, when the value of electric to magnetic field ratio exceeds a certain critical value. Our theoretical results, strikingly different from the standard $2 d$ electron gas, are explained using a 'Lorentz boost', and as an 'instability of a relativistic quantum field vacuum'. It is a remarkable case of emergent relativistic type phenomena in non-relativistic graphene. We also discuss few possible experimental consequence.
\end{abstract}

Graphene has been in the forefront of nano electronics and quantum condensed matter physics in the last couple of years. The mechanical rigidity, being able to peel off single graphene layer [1] and ability to make electrical contacts has made the system very appealing from building devices and experimental points of view [2] [3]. One of the remarkable discoveries in graphene is the anomalous $2 d$ quantized Hall effect. A variety of rich physics and anomalous phenomena are tied to a remarkable 'relativistic like' spectrum that electron and holes possess in graphene [4] 5]. This has made graphene important and interesting from several points of view in physics.

In the present letter, we investigate the effect of a uniform electric field, applied along the graphene sheet, on its already anomalous Landau level spectrum. We find that, within the low energy approximation near the Fermi surface (Dirac points), the problem can be exactly solved. We find strikingly new effects of electric field on the Landau levels which is different from the Landau levels of standard $2 d$ electron gas.

We find that the Landau spectrum gets scaled, for a given $k_{y}$ quantum number, by an electric field dependent dimensionless parameter $\left(\beta=\frac{E}{v_{F} B}\right)$. As the value of this parameter is increased, spacings between the Landau levels decreases. This Landau level contraction is consequence of electric field induced quantum mechanical mixing of Landau levels. The entire Landau level structure collapses at a critical value of this parameter. Further, the 'relativistic' character of the spectrum (with Fermi velocity replacing the velocity of light), leads to a novel interpretation of our result in terms of relativistic boosts and the mixing of electric and magnetic fields in moving frames of reference. We confirm our analytical result by solving the full tight binding model for a graphene sheet in the presence of magnetic and electric fields numerically. The collapse seen in the low energy approximation, is indeed accelerated in the actual tight binding model.

The modified wave functions and energy spectrum will have implications on the nature of quantum Hall break down. We briefly touch upon this issue at the end, and point out how it could be different from the standard quantum Hall break down.

Electronic states of graphene are well described by the tight binding hamiltonian for the $\pi$ electrons of the carbon atoms. In graphene, the carbon atoms form a triangular lattice with a basis of two geometrically inequivalent atoms placed $\frac{a}{\sqrt{3}}$ apart, where $a=2.456 A^{0}$ is the lattice constant. The overlap integral between the nearest carbon atoms is $t \approx 2.71 \mathrm{eV}$. We denote the triangular lattice sites by $\mathbf{R}_{i}=i_{1} \hat{\mathbf{e}}_{1}+i_{2} \hat{\mathbf{e}}_{2}$, where $\hat{\mathbf{e}}_{1}=\hat{\mathbf{x}}$ and $\hat{\mathbf{e}}_{2}=-\frac{1}{2} \hat{\mathbf{x}}+\frac{\sqrt{3}}{2} \hat{\mathbf{y}}$ are the basis vectors. $c_{\mathbf{i} r \sigma}(r=1,2$ and $\sigma=\uparrow, \downarrow)$ represent the electron annihilation operators with sub-lattice index $r$ and spin index $\sigma$ at $\mathbf{R}_{i}$. The hamiltonian is then written as,

$$
H=-t \sum_{\mathbf{i} \sigma} c^{\dagger} \mathbf{i} 2 \sigma\left(c_{\mathbf{i} 1 \sigma}+c_{\mathbf{i}+\hat{\mathbf{e}}_{2} 1 \sigma}+c_{\mathbf{i}-\hat{\mathbf{e}}_{3} 1 \sigma}\right)+h . c .
$$

where $\hat{\mathbf{e}}_{3}=-\left(\hat{\mathbf{e}}_{1}+\hat{\mathbf{e}_{2}}\right)$. The electronic dispersion for graphene has two points in a Brillouin zone which separates the positive and negative energy eigenstates. These so called Dirac points are $K_{1,2}= \pm \frac{2 \pi}{a}\left(\frac{1}{\sqrt{3}} \hat{\mathbf{x}}+\hat{\mathbf{y}}\right)$. The dispersion relation in the proximity of the Dirac points is linearly proportional to $|\mathbf{k}|$. The low energy modes around these points are described by slowly varying fields $\psi_{r \eta \sigma}\left(\mathbf{R}_{i}\right)$ defined as,

$$
c_{i r \sigma}=e^{i \mathbf{K}_{1} \cdot \mathbf{R}_{i}} \alpha_{r r^{\prime}}^{z} \psi_{r^{\prime} 1 \sigma}\left(\mathbf{R}_{i}\right)+e^{i \mathbf{K}_{2} \cdot \mathbf{R}_{i}} \alpha_{r r^{\prime}}^{x} \psi_{r^{\prime} 2 \sigma}\left(\mathbf{R}_{i}\right)
$$

Where $\alpha^{x}, \alpha^{y}$ are the Pauli matrices. The effective hamiltonian for the low energy modes is the Dirac hamiltonian.

$$
H=v_{F} \int d^{2} x \sum_{\eta \sigma} \Psi_{\eta \sigma}^{\dagger} \boldsymbol{\alpha} \cdot \mathbf{p} \Psi_{\eta \sigma}
$$

where $v_{F}=\frac{\sqrt{3}}{2} \frac{a t}{\hbar}$ is the Fermi velocity. $\Psi_{\eta \sigma}$ are two component field operators where $\eta(=1,2)$ is the valley index, corresponds to two Dirac points and $\sigma(=\uparrow, \downarrow)$ is the spin index. The spectrum can be obtained by solving the one particle equation to get the linear dispersion, $\epsilon(\mathbf{k})= \pm \hbar v_{F}|\mathbf{k}|$. In presence of an external magnetic field perpendicular to the graphene plane the one particle hamiltonian, $h=v_{F} \boldsymbol{\alpha}$. $\boldsymbol{\Pi}$, where $\boldsymbol{\Pi}=\mathbf{p}+e \mathbf{A}$, The energy eigenvalues are

$$
\epsilon_{n, k_{y}}=\operatorname{sgn}(n) \sqrt{2|n|} \frac{\hbar v_{F}}{l_{c}}
$$




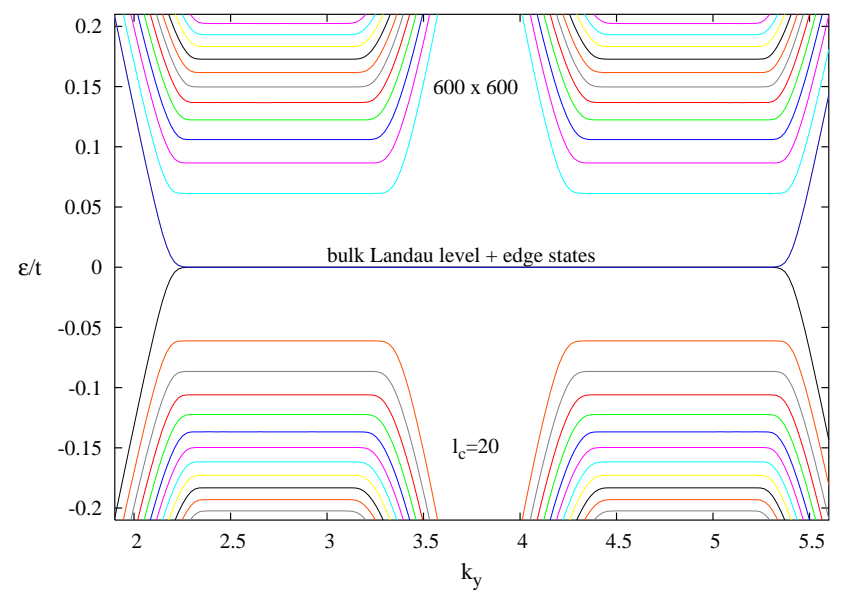

FIG. 1: Energy eigenvalues $\epsilon_{n, k_{y}}$, for electrons in graphene computed from the tight binding model for a hexagonal lattice subjected to a magnetic field $B=27.3$ Tesla (or $l_{c}=20 a$ where $a$ is the triangular lattice spacing) for a system size of $600 a \times 600 a$. The plot shows $\epsilon_{n, k_{y}}$ in the units of $t$ as function of $k_{y}$, where $k_{y}$ is the wavevector in the $y$ direction. Two sets of horizontal lines are Landau levels corresponding to the two valleys and $n=0$ Landau level and the edge states are degenerate

$n$ is the Landau level index, $k_{y}=\frac{2 \pi}{L_{y}} l$ is the quantum number corresponding to translation symmetry along $y$ axis, both $n$ and $l$ are integers (we choose Landau gauge $\mathbf{A}(\mathbf{r})=x B \hat{\mathbf{y}})$ and $l_{c}=\sqrt{\frac{\hbar}{e B}}$ is the magnetic length. Unlike the case of the non-relativistic electron in a magnetic field, where the spectrum has a linear dependence on the magnetic field and the non-negative integer valued Landau level index, the graphene Landau levels have a square root dependence on both magnetic field and Landau level index. The degeneracy of each level is given by the number of magnetic flux quanta passing through the sample. The eigenfunctions are,

$$
\psi_{n k_{y}}(x, y) \propto e^{i k_{y} y}\left(\begin{array}{c}
\operatorname{sgn}(n) \phi_{|n|-1}(\xi) \\
i \phi_{|n|}(\xi)
\end{array}\right)
$$

where $\phi_{n}(\xi)$ are the harmonic oscillator eigen-functions and $\xi \equiv \frac{1}{l_{c}}\left(x+l_{c}^{2} k_{y}\right)$.

We now consider the above system in the presence of a constant electric field in the $x$-direction. The single particle hamiltonian is then given by,

$$
h=v_{F} \boldsymbol{\alpha} \cdot \boldsymbol{\Pi}+\mathbf{1} e E x
$$

The Lorentz covariant structure of the hamiltonian, with $v_{F}$ playing the role of the speed of light, can be used to solve it exactly [6]. It is known from special relativity, if $v_{F} B>|\mathbf{E}|$, then we can always boost to a frame of reference where the electric field vanishes and the magnetic field is reduced. We can then use the solution in Eq.(4) and boost back to get the exact spectrum of the hamiltonian in Eq.(6). Here the boost transformation

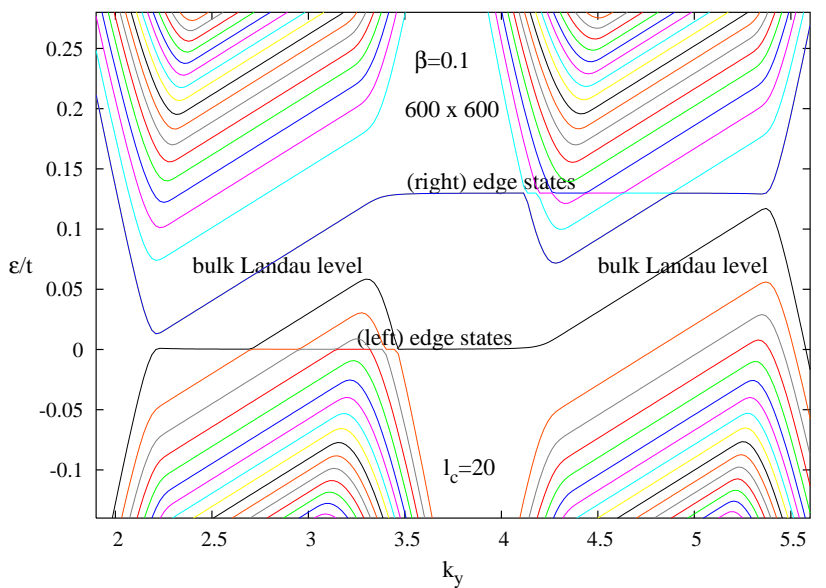

FIG. 2: Energy eigenvalues $\epsilon_{n, k_{y}}$ for electrons computed for the tight binding model for parameters given in Fig 1 and an external electric field $E$ applied along $x$-axis, given by the parameter $\beta=\frac{E}{v_{F} B}=0.1$. The electric field gives a linear $k_{y}$ dependence to the bulk Landau levels whereas it gives a constant shift to the edge states. The part of solid line labelled 'bulk Landau level' are $n=0$ Landau levels and parallel lines above and below them are Landau levels corresponding to positive and negative $n$ respectively. Set of points parallel to $k_{y}$ labelled 'edge states' are surface states localised at the zig-zag boundary.

amounts to doing a transformation on the space-time coordinate system. To implement the above procedure, it is convenient to work with the manifestly covariant time dependent Dirac equation,

$$
i \hbar \gamma^{\mu}\left(\partial_{\mu}+i \frac{e}{\hbar} A_{\mu}\right) \Psi\left(x^{\mu}\right)=0
$$

Where $x^{0}=v_{F} t, x^{1}=x, x^{2}=y, \gamma^{0}=\alpha^{z}, \gamma^{1}=i \alpha^{y}$, $\gamma^{2}=-i \alpha^{x}, \partial_{\mu}=\frac{\partial}{\partial x^{\mu}} . A^{0}=\phi$, the scalar potential, $A^{1}=A_{x}, A^{2}=A_{y}$ and $\Psi\left(x^{\mu}\right)$ is a two component spinor. We now apply a Lorentz boost in the $y$-direction (perpendicular to the electric field),

$$
\left(\begin{array}{c}
\tilde{x}^{0} \\
\tilde{x}^{2}
\end{array}\right)=\left(\begin{array}{cc}
\cosh \theta & \sinh \theta \\
\sinh \theta & \cosh \theta
\end{array}\right)\left(\begin{array}{c}
x^{0} \\
x^{2}
\end{array}\right)
$$

and $\tilde{x}^{1}=x^{1}$. The wave function transforms, $\tilde{\Psi}\left(\tilde{x}^{\mu}\right)=$ $e^{\frac{\theta}{2} \alpha_{y}} \Psi\left(x^{\mu}\right)$. Applying the above transformations and choosing $\tanh \theta=\frac{E}{v_{F} B}=\beta$, we can rewrite the Dirac equation in Eq.(17),

$$
\left(\gamma^{0} \tilde{\partial}_{0}+\gamma^{1} \tilde{\partial}_{1}+\gamma^{2}\left(\tilde{\partial}_{2}+\frac{i}{l_{c}^{2}} \sqrt{1-\beta^{2}} \tilde{x}^{1}\right)\right) \tilde{\Psi}\left(\tilde{x}^{\mu}\right)=0(9)
$$

In the boosted coordinates, where $|\beta|<1$, it is a problem of a Dirac electron in a (reduced) magnetic field, $\tilde{B}=$ $B \sqrt{1-\beta^{2}}$. The time component of the 3 -momentum in the boosted frame, $\tilde{\epsilon}_{n, \tilde{k}_{y}}=\operatorname{sgn}(n) \sqrt{2|n|} \frac{\hbar v_{F}}{l_{c}}\left(1-\beta^{2}\right)^{\frac{1}{4}}$ is not the physical energy eigenvalue of our problem. We 
have to apply the inverse boost transformation to obtain the spectrum and eigenfunctions of our problem,

$$
\begin{gathered}
\epsilon_{n, k_{y}}=\operatorname{sgn}(n) \sqrt{2|n|} \frac{\hbar v_{F}}{l_{c}}\left(1-\beta^{2}\right)^{\frac{3}{4}}-\hbar v_{F} \beta k_{y} \\
\Psi_{n, k_{y}}(x, y) \propto e^{i k_{y} y} e^{-\frac{\theta}{2} \alpha_{y}}\left(\begin{array}{c}
\operatorname{sgn}(n) \phi_{|n|-1}\left(\xi^{\prime}\right) \\
i \phi_{|n|}\left(\xi^{\prime}\right)
\end{array}\right) \\
\xi^{\prime} \equiv \frac{\left(1-\beta^{2}\right)^{\frac{1}{4}}}{l_{c}}\left(x+l_{c}^{2} k_{y}+\operatorname{sgn}(n) \frac{\sqrt{2|n|} l_{c} \beta}{\left(1-\beta^{2}\right)^{\frac{1}{4}}}\right)
\end{gathered}
$$

The energy eigenvalues of the standard $2 d$ electron gas in crossed magnetic and electric fields are given by $\epsilon_{n, k_{y}}=\left(n+\frac{1}{2}\right) \hbar \omega_{c}-\hbar k_{y} \frac{E}{B}-\frac{m}{2}\left(\frac{E}{B}\right)^{2}$. The main difference between the two besides the $\sqrt{n}$ and $\sqrt{B}$ dependence, is that the low lying graphene Landau level spacing scales as $\left(1-\beta^{2}\right)^{\frac{3}{4}}$, whereas the spacing is independent of the electric field in the non-relativistic case. Comparing the eigenfunctions with and without the electric field (11] 5), we see that the effect of the electric field is to (un)squeeze the oscillator states as well as to mix the particle and hole wave-functions. Squeezing corresponds to the change in $l_{c}$ and the eigenfunctions in Eq.(11) can be expanded as superposition of states in Eq.(5). Thus, unlike in the usual semiconductor samples, in graphene the electric field causes Landau level mixing. Also notice in (12) that the location of the gaussian also shifts as a function of the Landau level index $n$, unlike the standard $2 d$ electron gas.

As $\beta$ approaches unity, from Eq.(12) we infer that, to keep the gaussian shifts within the linear extent of the system requires larger values of $k_{y}$, which takes us beyond the long wavelength approximation. Moreover the Eq.(10) hands a collapse of the Landau level spectrum at $\beta=1$. One may wonder if the collapse we have found is an artifact of the low energy approximation? Interestingly, we find that in our full tight binding calculation the collapse persists, and infact it occurs at a value of $\beta$ even smaller than unity.

We have performed extensive numerical computations on the tight binding model for graphene with magnetic and electric fields, using lattice sizes ranging from $60 \times 60$ to $600 \times 600$. The magnetic field enters through the Peierls substitution, $t \rightarrow t e^{i \frac{2 \pi e}{\hbar} \int \mathbf{A} . d \mathbf{l}}$ in Eq (1). $\mathbf{A}(\mathbf{r})$ is chosen in such a way that the contribution to the phase term comes from hopping along one of the three bonds for each carbon atom. This enables us to maintain translation symmetry along the $\hat{\mathbf{e}}_{2}$ axis of the triangular lattice. The problem then reduces to the 1D Harper equation.

$$
\begin{aligned}
\epsilon \phi_{1, n_{1}} & =2 t \cos \left(\frac{k_{2} a+n_{1} \varphi}{2}\right) \phi_{2, n_{1}}+t \phi_{2, n_{1}+1} \\
\epsilon \phi_{2, n_{1}} & =2 t \cos \left(\frac{k_{2} a+n_{1} \varphi}{2}\right) \phi_{1, n_{1}}+t \phi_{1, n_{1}-1}
\end{aligned}
$$
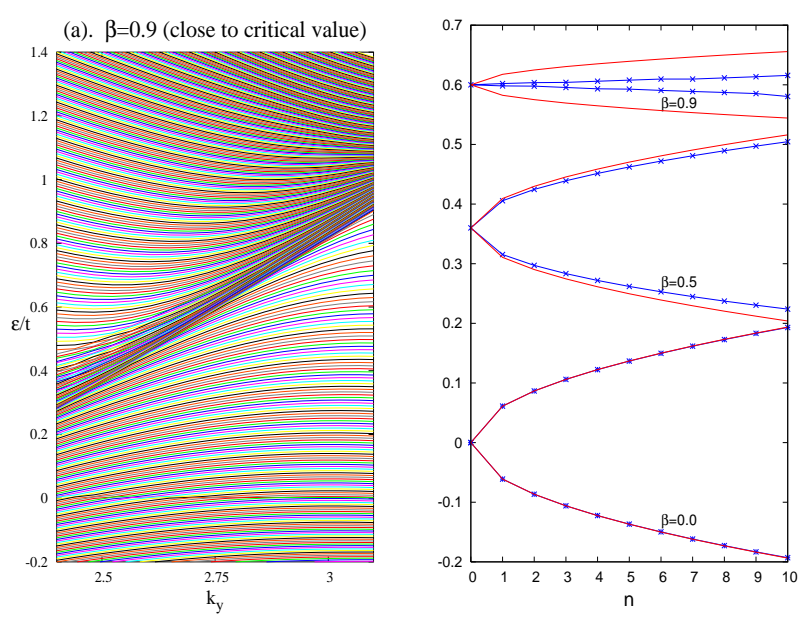

FIG. 3: (a) shows the energy eigenvalues around the Dirac point plotted as function of $k_{y}$ for $\beta=0.9$. The collapse can be clearly seen (b) shows the modulus of the eigenvalues $\left|\epsilon_{n, k_{y}}\right|$ for a value of $k_{y}=2.785$ computed from the tight binding model for system size $600 a \times 600 a$, magnetic field $B=27.3$ Tesla or $l_{c}=20 a$, electric field given by parameter $\beta=0.0,0.5,0.9$, plotted as a function of $n$.

Here $\varphi$ is the magnetic flux passing through each plaquette, $k_{2}$ is the wave vector and $n_{1}$ is the $\hat{\mathbf{e}}_{1}$ component of triangular lattice coordinate.

We choose the value of the magnetic field such that $L \gg l_{c} \gg a$, where $L$ is the linear extent of the system. The condition $l_{c} \gg a$ ensures that we stay away from the Hofstadter butterfly kind of commensurability effects on the spectrum and $L \gg l_{c}$ ensures that a large number of cyclotrons orbits fit in the sample. For our numerics, we expressed all energies in units of $t$ and all lengths in units of $a$.

Fig.(11) shows the results of our numerical investigation for zero and Fig.(2) for a finite $(\beta=0.1)$ electric fields. Fig.(11) shows the spectrum at low energies and the eigenvalues that are constant w.r.t $k_{y}$ are the Landau levels. They have $\sqrt{n}$ behaviour and are in excellent agreement with the analytical result and the eigenstates that vary with $k_{y}$ are the chiral edge states responsible for the quantum Hall current. In our numerics the lattice has zig-zag edges at the two ends along $\hat{\mathbf{e}}_{1}$. It is well known that the zig-zag edges result in zero-eigenvalue states even in the absence of external magnetic field [7]. These zero-eigenvalues surface states which are localised at the boundary and $n=0$ Landau level forms degenerate set of states as shown in Fig.(1). However, Fig.(2) shows that this degeneracy gets lifted in presence of an electric field. For small electric fields the wavefuntion of these edge states continues to be localised near edge of the sample. Any difference in eigenvalues of these surface states is because of the potential seen by them due to the externally applied electric field at the two edges of 
the sample. A characteristic feature of these edge states is that they don't vary with the wavevector, whereas the Landau levels develop a linear $k_{y}$ dependence with electric field.

Fig.(3b), shows $\sqrt{n}$ scaling of Landau levels for a given $k_{y}$ value. For zero electric field we see an excellent match between analytics and numerics. And for the case of finite electric fields we see a systematic deviation from exact results as we suspected from our exact result. As $\beta \rightarrow 1$, the tight binding results shows a faster collapse. Fig.(3 3 a) shows the collapse has already occurred at $\beta=0.9$, near one the Dirac points.

We show below that one of the consequences of the Landau level contraction (10) and the $n$ dependent guassian shift (12) is the possibility of a 'dielectric breakdown', which is different from the conventional ones. The single particle spectrum and states we have obtained thus far (for a given $E$ and $B$ ) can be used to construct stable many-body quantum Hall ground states. However, the external electric field not only modifies the single particle wave function and spectrum, but can also destabilise the ground state through spontaneous creation of particlehole pairs; i.e., by a dielectric breakdown. We present a simple formula for dielectric breakdown, without giving full details. It has an unusual dependence on the length scale over which the potential fluctuates and on the Landau level index $n$. This peculiar feature is absent in standard quantum $2 d$ electron systems [8]. Specifically we find that for slowly varying electric field fluctuations over a length scale $\ell_{E}$ and for large Landau level index, the critical voltage for breakdown is given by:

$$
V_{c} \approx \frac{\Delta_{n}(0, B)}{e}\left(1 \pm \kappa n\left(\frac{l_{c}}{\ell_{E}}\right)^{2}\right)
$$

where $\kappa$ is a constant of the order of unity, which depends on the strength of the electric field fluctuations and $\Delta_{n}$ is gap between levels $n$ and $n+1$. This means that if we have an electric field, non-uniform over a nanoscopic scale $\left(\ell_{E} \sim l_{c}\right)$, it will cause local breakdown even before the critical field is reached. Such situations can be created through in plane or out of plane charged impurities or STM tips, in addition to external electric fields. It is interesting that such an anomalous local breakdown is Landau level index $n$ dependent, we expect that the quantum Hall breakdown should be qualitatively different for $n=0$ and $n \neq 0$ within graphene.

In the light of new spectroscopic experiments 9], we claim that the contraction in Landau level spacing and the collapse can be observed at fields attainable in laboratories. The gap between $n=0$ and $n=1$ for $B \sim 1$ Tesla is $\sim 35 \mathrm{meV}$, for $E \sim 3 \times 10^{5} \mathrm{Vm}^{-1}, 10 \%$ reduction in the gap is expected. And the collapse of the Landau levels should also be observeable by applying $E \sim 10^{6} \mathrm{Vm}^{-1}$. In the context of quantum Hall breakdown, the dependence of critical voltage on $\ell_{E}$ as given in Eq.(14) suggests that the breakdown phenomena should be different from what we observe in standard $2 d$ quantum Hall system. Moreover graphene's Landau level index dependence on $V_{c}$, we expect the breakdown phenomena is going to be different for $n \neq 0$ from that of $n=0$.

It will be interesting to study graphene from the point of view of the present paper. As quantum Hall phenomena are beginning to be seen in pyrolytic graphite 10] and possibly in carbon eggshells [11], it will be very interesting to study electric field effects in these systems as well, to confirm our predictions.

In summary, we have made a theoretical prediction of a remarkable phenomena in graphene: Landau level contraction and an eventual collapse, induced by crossed electric fields. The local dielectric breakdown has a peculiar length and Landau level index dependence. These phenomena, not known in the standard $2 d$ electron gas, is a consequence of the relativistic type spectrum of low energy electrons and holes in graphene.

* Electronic address: vinu@imsc.res.in

† Electronic address: shankar@imsc.res.in

‡ Electronic address: baskaran@imsc.res.in

[1] K.S. Novoselov et al., Science, 306, 666(2004); C. Berger et al., J. Phys. Chem., 108, 19912 (2004)

[2] K.S. Novoselov et al., Nature 438,197(2005); Y. Zhang et al., Nature 438,201(2005); Y. Zhang et al., Phys. Rev. Lett. 96, 136806 (2006)

[3] K.S. Novoselov et al.,Nature Physics 2, 177-180(2006)

[4] V.P. Gusynin, S.G. Sharapov, Phys.Rev.Lett. 95 146801(2005); C.L. Kane, E.J. Mele, Phys. Rev. Lett. 95 146802(2005); ibid 95, 226801(2005); A. H. Castro Neto, F. Guinea, N. M. R. Peres, Phys.Rev.B 73, 205408 (2006); M. I. Katsnelson, cond-mat/0512337; J. Tworzydlo et al. cond-mat/0603315; D.V.Khveshchenko, cond-mat/0602398 $\quad$ N. A. Sinitsyn et al. cond-mat/0602598, D. N. Sheng, L. Sheng, Z. Y. Weng cond-mat/0602190, N. M. R. Peres, F. Guinea, A. H. Castro Neto, Phys.Rev. B 73, 125411(2006); ibid 72, 174406(2005);ibid. 73,125411 (2006) cond-mat/0512476 cond-mat/0506709, cond-mat/0603155

[5] E.McCann, V. I. Falko, Phys.Rev.Lett.96, 086805(2006); J. Nilsson et al cond-mat/0512360,

[6] A.H. MacDonald, Phys. Rev. B 28, 2235(1983)

[7] Y. Niimi et al., Phys. Rev. B 73, 085421(2006); L. Brey, H.A. Fertig, cond-mat/0602505 cond-mat/0603107. K. Sasaki, S. Murakami, R. Saito, cond-mat/0602647; A. Abanin, P. A. Lee, L. S. Levitov, Phys. Rev. Lett. 96, 176803(2006); D. N. Sheng, L. Sheng, Z. Y. Weng, cond-mat/0602190 V. M. Pereira et al, Phys. Rev. Lett. 96, 036801 (2006)

[8] G. Ebart et al., J. Phys. C 16, 5441(1983); V. Tsemekhman et al., Phys. Rev. B 55, R10201

[9] M.L. Sadowski et al., cond-mat/0605739

[10] H. Kempa, P. Esquinazi, Y. Kopelevich, cond-mat/0603155

[11] Timir Datta, et al cond-mat/0503166 